\title{
Free vibrations of thin-walled semi-underground main gas pipelines of large diameter, with the attached ground mass
}

\author{
Vladimir Sokolov ${ }^{1}$ and Igor Razov ${ }^{1, *}$ \\ ${ }^{1}$ Industrial University of Tyumen, Volodarskogo str. 38, Tyumen, 625001, Russia
}

\begin{abstract}
In this paper, we solve the problem of free bending vibrations of the main thin-walled large-diameter pipeline, corresponding semiunderground laying type. As a result, vibration process involves a pipeline to move the surrounding ground, which significantly affects the frequency free vibrations. The values of the natural frequencies of the pipeline will depend on the characteristics of the ground, and it's mass. The decision takes into account the impact of the attached ground mass on the free vibration frequency based on the geometrically nonlinear version of semimomental theory of average bending cylindrical shells.
\end{abstract}

\section{Introduction}

Pipeline transportation is a major component of the fuel and energy complex of the country, which has a length of more 10 thousand kilometers from the place of production to the consumer. At present, our country is implementing the construction of new and development of existing trunk pipeline networks made of modern thin-walled pipes with diameter up to $1000 \mathrm{~mm}$. Depending on the region, climatic and geological conditions there are different types of technological solutions used for laying pipelines such as underwater[1,2], above-ground, ground [3,4,5,6,7,8,9,10], underground $[11,12,13,14]$ and semi-underground $[15,16]$. For each type of laying there are the features of calculation and collecting of loads to be considered for exploitation. Semi-underground laying scheme (Fig. 1), [17] is used for pipeline crossings of waterlogged, the presence of the underlying rocks, as well as at the intersections with other communications. According to the production schedules of mound of the pipeline is made of mineral and ground hydrophobized. The purpose of this mound is to prevent freezing of insulation from the ground, de-aeration of the ground electrolyte, reducing the risk of biocorrosion etc.

As a result, vibration process, the ground surrounding the pipe involved in the movement, and has an additional effect on the vibrating system, that is to the forces of inertia vibrating pipe is added to the inertial effect of the so-called attached ground mass.

*Corresponding author: RazovIO@mail.ru 

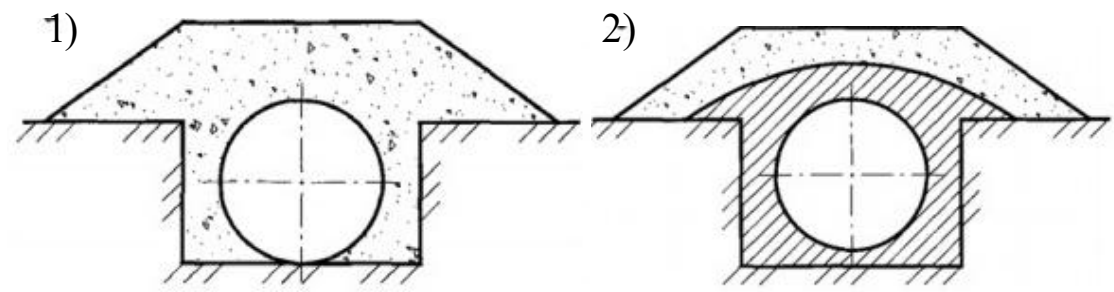

Fig. 1.Semi-underground pipeline laying, where 1 - mound of mineral ground; 2 - mound of ground water-repellent.

\section{Materials and methods}

Semi-momental theory of shell based on assumptions that founded the general case, A.L. Goldenweiser, and V.P. Ilyin [18]for the calculation of thin-walled tubes. In [18] as suggested the geometrically nonlinearversionof semi-momental theory of shells, which allows you to take into account the deformation of the cross-sectional area and the impact of the internal operating pressure on the frequency of free vibrations.

The pipe shells made of homogeneous, isotropic material with a modulus of elasticity E, Poisson'sratiov, and according to the hypothesis of the Kirchhoff - Love, the following assumptions are made:

1) Relative elongation $\varepsilon_{2}$ in the circumferential direction of the shell cross sections, defined by relation of the general theory of shells $\varepsilon_{2}=\frac{\partial v}{\partial \theta}+w$, assumed to be small compared with the derivative of the tangential movementvandwithradial movement $w$, that is use: $\frac{\partial v}{\partial \theta}+w=0$;

2) The relative shift of the middle surface of the shell $\widetilde{\omega}$ accepted to be small compared with angle of rotation in the cross and longitudinal directions: $\frac{\partial v}{\partial \xi}+\frac{\partial u}{\partial \theta}=0$;

3) Angle of rotation tangent to the cross-sectional middle line shell $\vartheta_{2}$, changing curvature in the longitudinal $\aleph_{1}$ and $\aleph_{2}$ cross direction, and the torsion of the middle surface of the shell $\tau$ are expressed through movement components $u, v, w$, through thegeneral relations of the theory of shells: $\vartheta_{2}=\frac{\partial w}{\partial \theta}-v, \aleph_{1}=-\frac{1}{R} \frac{\partial^{2} w}{\partial \xi^{2}}$, $\aleph_{2}=-\frac{1}{R} \frac{\partial \vartheta_{2}}{\partial \theta}, \varepsilon_{1}=\varepsilon_{0}+\frac{\partial u}{\partial \xi}, \tau=-\frac{1}{R} \frac{\partial \vartheta_{2}}{\partial \xi}, \varepsilon_{0}=\frac{F}{E A} ;$

4) The relations between the force and deformation are of the form [18]:

5) $T_{1}=E h \varepsilon_{1}, M_{2}=D \aleph_{2}, T_{2}=-\widetilde{v} E h \varepsilon_{1}, M_{1}=\widetilde{v} D \aleph_{2}$, shift force $S=\frac{E h}{2(1+v)} \widetilde{\omega}$;

6) The balance equations, the general theory of shells you can miss the value of across forces $Q_{1}$ and the value of torque $H$, in the last equation of the moments as it is accepted in the work [18].

The following designations are accepted: $M_{1}$ and $M_{2}$ - bending moments in the longitudinal and cross (circular) directions; $T_{1}$ and $T_{2}$ - longitudinal and circumferential 
tangential forces; $\aleph_{2}$ - change the coordinate lines of curvature $\theta$ middle surface; $\varepsilon_{1}$ and $\varepsilon_{2}-$ the relative elongation in the direction orthogonal dimensionless coordinates $\xi=x / R$ and $\theta$, where $x$ - coordinate along the longitudinal axis of the shell; $\tau$ - twist the middle surface along of the shell; $D=\frac{E h^{3}}{12\left(1-v^{2}\right)}-$ the cylindrical rigidity. $u, v, w$-related to the radius $R$ tangentially (along the coordinates $\left.\xi_{\text {и }} \theta\right)$ andradial movement of the middle surface of the shell.

For receive the equation we use the assumptions of geometrically nonlinear version of semi-momental theory of shells of Galimov-Mushtari[19], according to which the greatest displacement value comparable with thickness $h$, but small compared with the radius of middle surface $R$. The squares of the rotation angles accepted small compared with one:

$$
1+\frac{1}{2} \vartheta_{2}^{2} \approx 1,1+\frac{1}{2}\left(\frac{\partial w}{\partial \xi}\right)^{2}+\frac{1}{2}\left(\frac{\partial v}{\partial \xi}\right)^{2} \approx 1
$$

Where $\vartheta_{2}$ - the angle of rotation of the tangent to middle line cross-sectional, and the curvature of the deformed cross-section of the shell is determined by the equation:

$$
\frac{1}{R_{2}^{*}}=\frac{1}{R}+\aleph_{2}=\frac{1}{R}\left(1-\frac{\partial \vartheta_{2}}{\partial \theta}\right)
$$

Where $R_{2}$ - the radius of curvature of middle line of the cross section deformed stage of shell.

The curvature of the shell in the deformed stage in the longitudinal direction:

$$
\frac{1}{R_{1}^{*}}=-\frac{1}{R} \frac{\partial^{2} w}{\partial \xi^{2}}
$$

In view of the assumptions $1-5$ andLame parameter values $A_{1}=1, A_{2}=$ $R$ forcylindrical shells in a deformed stage, we receive the equation of balance of the middle surface of the shell element, from the general equations of the nonlinear theory of shells middle bend Galimov - Mushtari[19]:

$$
\begin{aligned}
& \frac{\partial T_{1}}{\partial \xi}+\frac{\partial S}{\partial \theta}+R Q_{2} \tau=-R X_{1}, \\
& \frac{\partial T_{2}}{\partial \theta}+\frac{\partial S}{\partial \xi}+\frac{R}{R_{2}^{*}} Q_{2}=-R X_{2}, \\
& \frac{\partial Q_{2}}{\partial \theta}-\frac{R}{R_{2}^{*}} T_{2}-\frac{R}{R_{1}^{*}} T_{1}=-R X_{3}, \\
& \frac{\partial M_{1}}{\partial \xi}+\frac{\partial H}{\partial \theta}-R Q_{1}=0 \\
& \frac{\partial M_{2}}{\partial \theta}-\frac{\partial H}{\partial \xi}-R Q_{2}=0
\end{aligned}
$$

where, forces and moments per unit length of the section marked with the index 1, acting in the longitudinal direction, marked 2 - in the annular; $T_{i}$ - normal forces, $Q_{i}-$ cross forces, $S_{1}=S_{2}=S_{-}$shift forces, $M_{\mathrm{i}}-$ bending moment, $X_{1}, X_{2}, X_{3}-$ tangential and normal components of external load. 
System of equations (3) it is a system of geometrically nonlinear differential equations, because have nonlinear term with torsion $\tau$ in the first equation of the system, and also curvature $1 / R_{2}^{*}$ и $1 / R_{1}^{*}$ in the third equation of the system there are curvature of shellin the deformed stage.

\section{Results and discussion}

Dynamic calculation of thin-walled pipes, and now days is an actual problem, which confirms a series of works devoted to this problem [2-16,18]. Practice shows that the pipelines during operation are subjected to dangerous kind of dynamic effects and vibrations. Long exposure of increased vibration in combination with other factors may cause fatigue destruction of the pipeline elements. So to perform dynamic calculation is necessary to know the dynamic characteristics of the thin-walled main gas pipeline of large diameter, such as the spectrum of its own frequencies. Calculations executed by SNIP [20] and other normative documents, as a rule, are based on a rod theory, and involve some aspects of pipeline operation reliability. For this design scheme cannot account for the effect of some important factors specific to the real thin-walled pipes, for example, the cross-sectional deformation and the influence of the internal operating pressure on the dynamic characteristics and the stability of the pipeline. So for the calculation of thinwalled pipes of large diameter design scheme taken in the form of a thin cylindrical shell of finite length $\mathrm{L}$, middle surface radius $\mathrm{R}$, and wall thickness $\mathrm{h}$. This shell is exposed to the action of a constant internal operating pressure $p_{0}$, the influence of inhomogeneous elastic resistance on the ground on cross-section of shell and longitudinal compressive forces $F$.Inhomogeneous elastic impact resistance of ground on the cross-section of the pipeline in the first approximation is described by the equation (Figure 2):

$$
q_{k}=k R w(1-\alpha \cos \theta),
$$

where $q_{k}$-ground pressure to the external tube wall; $k$ - stiffness coefficient of elastic space, $\left[\mathrm{H} / \mathrm{M}^{3}\right], \alpha-$ coefficient depending on the ground characteristics, determined experimentally for each specific case.

As a result, vibration process the ground surrounding the pipeline is included in the movement of the entire system, and is called the adjoined mass of ground. This effect more related to water saturated grounds in boggy areas.

Attached mass of ground involving a gas pipeline under the vertical elastic vibrations determined by the equation[21]:

$$
\mu_{2 p j}=k_{2 p j} \mu_{2 p}
$$

where $k_{\text {zpj }}$ - correction factor, which depends on the tone number of vibrations ( $\mathrm{j}-$ tone number of vibrations), $\mu_{2 p}$-attached mass of ground per unit length of the pipeline.

$$
\mu_{z p}=\frac{\gamma_{z p} \pi d^{2}}{4},
$$

where $\gamma_{2 p}$ - density of the surrounding ground of gas pipeline; $\mathrm{d}-$ the outer diameter of the pipeline. 


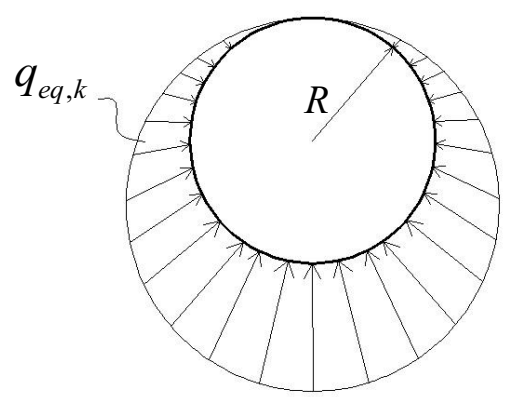

Fig. 2.Inhomogeneous distribution of ground pressure on the perimeter of the cross section of the gas pipeline.

Based on the a geometrically nonlinear version of semi-momental theory of cylindrical shells, are in the deformed stage, the motion equation is:

$$
\begin{gathered}
\frac{\partial^{2} T_{1}}{\partial \xi^{2}}+\frac{\partial}{\partial \xi}\left(\tau \frac{\partial M_{2}}{\partial \theta}\right)-\frac{\partial^{2}}{\partial \theta^{2}}\left(\frac{R_{2}^{*}}{R_{1}^{*}} T_{1}\right)-\frac{1}{R^{2}} \frac{\partial^{2}}{\partial \theta^{2}}\left(R_{2}^{*} \frac{\partial^{2} M_{2}}{\partial \theta^{2}}\right)- \\
-\frac{\partial}{\partial \theta}\left(\frac{1}{R_{2}^{*}} \frac{\partial M_{2}}{\partial \theta}\right)+R \frac{\partial X_{1}}{\partial \xi}-R \frac{\partial X_{2}}{\partial \theta}-\frac{\partial^{2}}{\partial \theta^{2}}\left(R_{2}^{*} X_{3}\right)=0
\end{gathered}
$$

where $X_{2}=-R h \rho \frac{\partial^{2} v}{\partial t^{2}}, X_{3}=-R h \rho \frac{\partial^{2} w}{\partial t^{2}}+\mu_{z p j} \frac{\partial^{2} w}{\partial t^{2}}+p_{0}-k R w(1-\alpha \cos \theta)$, $\rho$ - the density of the shell material.

To move to the solution of the problem in displacements have basic relations semimomental shell theory:

$$
T_{1}=E h \varepsilon_{1}, M_{2}=D \chi_{2}
$$

where $E$ - the elastic modulus of the shell material; $\varepsilon_{1}$ - longitudinal deformation repose in two parts: $\varepsilon_{1}=\varepsilon_{0}+\frac{\partial u}{\partial \xi}, \varepsilon_{0}=\frac{F}{E A}$.

The initial deformation $\varepsilon_{0}$ determined on the assumption of non-deformable crosssections of the shell, $A$ - cross sectional area. form:

The relations between deformations and displacements semi-momental theory have the

$$
\frac{\partial v}{\partial \theta}+w=0 ; \quad \frac{\partial v}{\partial \xi}+\frac{\partial u}{\partial \theta}=0 ; \quad \vartheta_{2}=\frac{\partial w}{\partial \theta}-v ; \tau=-\frac{1}{R} \frac{\partial \vartheta_{2}}{\partial \xi}, \chi_{2}=-\frac{1}{R} \frac{\partial \vartheta_{2}}{\partial \theta} .
$$

Substituting the relations (8), (9) into the equation (7), after some transformations, and discarding the nonlinear terms we get the linearized equation of shell in displacements: 


$$
\begin{gathered}
\frac{\partial^{3} u}{\partial \xi^{3}}+h_{v}^{2} \frac{\partial^{3}}{\partial \theta^{3}}\left(\vartheta_{2}+\frac{\partial^{2} \vartheta_{2}}{\partial \theta^{2}}\right)+2 \frac{\partial^{2}}{\partial \theta^{2}}\left(\frac{\partial^{2} w}{\partial \xi^{2}} \varepsilon_{0}\right)-\frac{R}{E h} p_{0} \frac{\partial^{3} \vartheta_{2}}{\partial \theta^{3}}+\mu_{\text {cpj }} \frac{\partial^{4} w}{\partial \theta^{2} \partial t^{2}} \frac{R}{E h}+ \\
+\frac{R^{2} k}{E h} \frac{\partial^{2} w}{\partial \theta^{2}}-\frac{R^{2} k \alpha}{E h} \frac{\partial^{2} w}{\partial \theta^{2}} \cos \theta+\frac{2 k R^{2}}{E h} \frac{\partial w}{\partial \theta} \sin \theta+\frac{k R^{2} \alpha w \cos \theta}{E h}- \\
-\frac{R^{2} \rho}{E}\left(\frac{\partial^{3} u}{\partial \xi \partial t^{2}}-\frac{\partial^{2} v}{\partial \theta \partial t^{2}}-\frac{\partial^{4} w}{\partial \theta^{2} \partial t^{2}}\right)=0
\end{gathered}
$$

Where $u, v, w$ - Components movement the middle surface of the shell, divided by the radiusR; $h_{v}$ - parameter of the relative thickness of the shell: $h_{v}=\frac{h}{R \sqrt{12\left(1-v^{2}\right)}}$, where $v$ Poisson's ratio.

The obtained system of equations (10) it contains four unknown functions of coordinates and timet: $u, v$, wand $\vartheta_{2}$. Solving this system, the variables separation method (Fourier method), we represent the function $w(\xi, \theta, t)$ in the case of hinged support end of shell, obeying the condition of periodicity on the circumferential coordinate $\theta$ :

$$
w=\sum_{m} \sum_{n} f(t) \sin \left(\tilde{\lambda}_{n} \xi\right) \cos (m \theta)
$$

where $\tilde{\lambda}_{n}=\frac{n \pi R}{L} ; m, n=1,2 \ldots$ - wave numbers in the circumferential and longitudinal directions. The other components of displacement and rotation angle of the tangent determined from the relations semi-momental shell theory (9):

$$
\begin{aligned}
& u=-\sum_{m} \sum_{n} \frac{\tilde{\lambda}_{n}}{m^{2}} f(t) \cos \left(\lambda_{n} \xi\right) \cos (m \theta), \quad v=-\sum_{m} \sum_{n} \frac{1}{m} f(t) \sin \left(\tilde{\lambda}_{n} \xi\right) \sin (m \theta), \\
& \vartheta_{2}=-\sum_{m} \sum_{n} \frac{m^{2}-1}{m} f(t) \sin \left(\tilde{\lambda}_{n} \xi\right) \sin (m \theta) .
\end{aligned}
$$

Assuming that free vibrations of a shell is harmonic, then we have:

$$
f(t)=\sin \omega_{m n} t, f^{\prime \prime}(t)=-\omega_{m n}^{2} \sin \omega_{m n} t,
$$

where $\omega_{m n}$-circular frequency of the shell on the form of the free bending vibrations $m, n=1,2,3 \ldots$.

Substituting (11) - (12) into the equation (10) and equating the coefficients of like trigonometric functions $\cos \mathrm{m} \theta$ at $\mathrm{m}=1,2,3 \ldots$, we obtain an infinite system of homogeneous linear algebraic equations for the unknown amplitude values $b_{m}$ of radial displacement componentw. The coefficients of the unknowns in these equations we denote $\mathrm{a}_{\mathrm{ij}}$, then:

$$
\begin{aligned}
& \text { whenm }=1 \quad a_{1,1} b_{1}+a_{1,2} b_{2}=0 \\
& \text { whenm }=2 \quad a_{2,1} b_{1}+a_{2,2} b_{2}+a_{2,3} b_{3}=0,(14) \\
& \text { whenm }=3 \quad a_{3,2} b_{2}+a_{3,3} b_{3}+a_{3,4} b_{4}=0
\end{aligned}
$$


The resulting homogeneous system of linear equations can be written in compact form:

$$
a_{m, m-1} b_{m-1}+a_{m, m} b_{m}+a_{m, m+1} b_{m+1}=0
$$

wherem $=1,2,3 \ldots \mathrm{m}-1>0$, andcoefficienta $\mathrm{i}_{\mathrm{ij}}$ is defined as:

$$
\begin{gathered}
a_{m, m}=A_{m n}-B_{m n} \omega_{m n}^{2} \\
a_{m, m \pm 1}=-\frac{m^{2}(m \pm 1)^{2}}{2} k^{*} \alpha \\
A_{m n}=\lambda_{n}^{4}+m^{4}\left(m^{2}-1\right)\left(m^{2}-1+p^{*}\right)+k^{*} m^{4}-\lambda_{n}^{4} m^{4} P / n^{2} \\
B_{m n}=\rho^{*} R \cdot h\left(\lambda_{n}^{2} h_{v}+m^{2}+m^{4}\right)+\mu_{z p j}^{*} m^{4}
\end{gathered}
$$

Where $p^{*}=p_{0} \frac{R}{E h h_{v}^{2}}, \rho^{*}=\rho_{0} \frac{R}{E h h_{v}^{2}}, k^{*}=\frac{R^{2} k}{E h h_{v}^{2}}, \mu_{\text {cpj }}^{*}=\mu_{\text {cpj }} \frac{R}{E h h_{v}^{2}}$.

The coefficients of the system of equations (15) are dimensionless in internal operating pressurep $_{0} \mathrm{inMPa}$, coefficient of elastic foundationkinN $/ \mathrm{M}^{3}$ and density of the shell material $\rho_{0}$ in $\frac{\kappa H}{M^{4}} c^{2}$.

For a detailed analysis of the resulting system of homogeneous linear algebraic equations (15) will present it in the form of a matrix:

$$
\left(\begin{array}{ccccc}
a_{1,1} & a_{1,2} & 0 & 0 & \ldots \\
a_{2,1} & a_{2,2} & a_{2,3} & 0 & \ldots \\
0 & a_{3,2} & a_{3,3} & a_{3,4} & \ldots \\
\ldots & \ldots & \ldots & \ldots & \ldots \\
\ldots & \ldots & \ldots & \ldots & \ldots
\end{array}\right)\left(\begin{array}{c}
b_{1} \\
b_{2} \\
b_{3} \\
\ldots \\
\ldots
\end{array}\right)=0 .
$$

Because the solution of the homogeneous system of linear algebraic equations is different from zero, as the value of the amplitude values of the radial movement of the middle surface of the shell $b_{m n} \neq 0$, determinant factors of the homogeneous system (17) should be equal to zero:

$$
\left|\begin{array}{ccccc}
a_{1,1} & a_{1,2} & 0 & 0 & \ldots \\
a_{2,1} & a_{2,2} & a_{2,3} & 0 & \ldots \\
0 & a_{3,2} & a_{3,3} & a_{3,4} & \ldots \\
\ldots & \ldots & \ldots & \ldots & \ldots \\
\ldots & \ldots & \ldots & \ldots & \ldots
\end{array}\right|=0 .
$$

Thus, the problem of determining the eigen frequenciesof a given straight section of the gas pipeline on the shell waveforms is proceed to a problem on the eigenvalues of the coefficients of the system of homogeneous linear algebraic equations (17). Then the determinant (18) takes the form of the characteristic equation of the $n$-th degree for a square matrix A $\mathrm{n}$ - th order:

Consider the truncated system of homogeneous linear algebraic equations obtained from (17) with $\mathrm{m}=1,2,3$. In matrix form, this system has the form: 


$$
A B=\left(\begin{array}{lll}
a_{1,1} & a_{1,1} & a_{1,1} \\
a_{1,1} & a_{1,1} & a_{1,1} \\
a_{1,1} & a_{1,1} & a_{1,1}
\end{array}\right) \times\left(\begin{array}{l}
b_{1} \\
b_{2} \\
b_{3}
\end{array}\right)=0
$$

The truncation of an infinite system of linear algebraic equations (17) does not significantly affect the accuracy of the solution of the problem, as this system is regular. A study conducted in according to the method showed that the sum of the moduls of the coefficients of minor members of each row of the matrix A divided by the coefficient module at the main diagonal terms, turns out to be less than one.

All the elements of the matrix are different from zero $\left(b_{m} \neq 0\right)$, therefore the determinant of the coefficient matrix A must be zero:

$$
|A|=\left|\begin{array}{lll}
a_{1,1} & a_{1,1} & a_{1,1} \\
a_{1,1} & a_{1,1} & a_{1,1} \\
a_{1,1} & a_{1,1} & a_{1,1}
\end{array}\right|=0
$$

The problem is determining the eigenfrequencies a given section of the pipeline is reduced to the problem of free values of the matrix A. Let the determinant (20) in the form of the characteristic equation of A. To this end, we write the determinant, substituting the values of the diagonal elements $a_{m, m}$ of the formulas (14):

$$
|A|=\left|\begin{array}{ccc}
A_{1 n}-B_{1 n} \omega_{1 n}^{2} & a_{1,2} & a_{1,3} \\
a_{2,1} & A_{2 n}-B_{2 n} \omega_{2 n}^{2} & a_{2,3} \\
a_{3,1} & a_{3,2} & A_{3 n}-B_{3 n} \omega_{3 n}^{2}
\end{array}\right|=0 .
$$

Multiply each row of the determinant on $\frac{1}{B_{m n}}$. As a result, we obtain:

$$
\frac{1}{B_{1 n} B_{2 n} B_{3 n}} \times\left|\begin{array}{ccc}
A_{1 n}-B_{1 n} \omega_{1 n}^{2} & a_{1,2} & a_{1,3} \\
a_{2,1} & A_{2 n}-B_{2 n} \omega_{2 n}^{2} & a_{2,3} \\
a_{3,1} & a_{3,2} & A_{3 n}-B_{3 n} \omega_{3 n}^{2}
\end{array}\right|=0 .
$$

Because the multiplier $\frac{1}{B_{1 n} B_{2 n} B_{3 n}}$, is different from zero, zero is equal to the determinant of which is the characteristic equation of the matrix $A$ :

$$
|A-\lambda E|=\left|\begin{array}{ccc}
d_{1,1}-\lambda & d_{1,2} & d_{1,3} \\
d_{2,1} & d_{2,2}-\lambda & d_{2,3} \\
d_{3,1} & d_{3,2} & d_{3,3}-\lambda
\end{array}\right|=0
$$

where the following notation:

$$
\lambda=\omega^{2}, \quad d_{m, m}=\frac{A_{m n}}{B_{m n}}, \quad d_{m, m \pm 1}=\frac{a_{m, m \pm 1}}{B_{m n}}
$$


Expanding the determinant (21), we obtain the characteristic equation of degree equal to the order of the matrix $\mathrm{A}$, whose roots $\lambda_{1}$ are called the eigenvalues determine the frequency of free vibrations of the squares of the considered section of the pipeline.

The characteristic equation of the matrix $A$ of order $k$ on the left side contains a polynomial of $\mathrm{k}$-th degree of $\lambda$ with leading coefficient equal to one, that degree of the characteristic equation are determined by the order of the matrix $\mathrm{A}$.

If we take the matrix of the 1 st order at a wave number $m=1$, then from (21) we obtain a linear characteristic equation for $\lambda$ :

$$
d_{1,1}-\lambda=0
$$

Substituting the corresponding values for (16) we obtain the equation:

$$
\omega_{1 n}^{2}=\frac{A_{1 n}}{B_{1 n}}
$$

Where for values of the coefficients of (16) we obtain the solution for the square of the frequency of free vibrations of the straight section of the pipeline at semi-underground laying with non-deformable contour of the cross section ( when $\mathrm{m}=1$ ):

$$
\omega_{m n}^{2}=\frac{\lambda_{n}^{4}+k^{*}-\lambda_{n}^{4} P / n^{2}}{2 \rho^{*} R \cdot h\left(\lambda_{n}^{2} h_{\widetilde{v}}\right)+\mu_{2 p j}^{*}}
$$

Where $\lambda_{n}=\frac{n \pi R}{L \sqrt{h_{\widetilde{v}}}}, p^{*}=p_{0} \frac{R}{E h h_{v}^{2}}, \rho^{*}=\rho_{0} \frac{R}{E h h_{v}^{2}}, \mu_{z p j}^{*}=\mu_{\text {zpj }} \frac{R}{E h h_{v}^{2}}, k^{*}=\frac{R^{2} k}{E h h_{v}^{2}}$.

In considering matrix 2 nd order at wave numbers $m=1$ and 2 , use (21) we obtain:

$$
\left|\begin{array}{cc}
d_{1,1}-\lambda & d_{1,2} \\
d_{2,1} & d_{2,2}-\lambda
\end{array}\right|=0
$$

then we get a square characteristic equation:

$$
\lambda^{2}-\left(d_{1,1}+d_{2,2}\right) \lambda+\left(d_{1,1} d_{2,2}-d_{1,2} d_{2,1}\right)=0,
$$

whose solution gives two roots $\lambda 1$ and $\lambda 2$, defining squares circular frequency of free vibrations of the pipeline section $\omega_{1 n}^{2}$ and $\omega_{2 n}^{2}$ of relevant forms of vibrations with $\mathrm{m}=1$ и 2 .

For a matrix $3 \mathrm{rd}$ order obtained at wave numbers $\mathrm{m}=1,2,3$, we come to the determinant (21), solve that, we obtain a cubic characteristic equation of the matrix A:

$$
\lambda^{3}-I_{1} \lambda^{2}+I_{2} \lambda-I_{3}=0
$$

constant coefficients have the form:

$$
\begin{gathered}
I_{1}=d_{1,1}+d_{2,2}+d_{3,3}, \\
I_{2}=d_{1,1} d_{2,2}+d_{2,2} d_{3,3}+d_{3,3} d_{1,1}-d_{1,2} d_{2,1}-d_{2,3} d_{3,2}-d_{3,1} d_{1,3},
\end{gathered}
$$




$$
I_{1}=d_{1,1} d_{2,2} d_{3,3}+d_{1,2} d_{2,3} d_{3,1}+d_{2,1} d_{3,2} d_{1,3}-d_{1,1} d_{2,3} d_{3,2}-d_{2,2} d_{3,1} d_{1,3}-d_{3,3} d_{2,1} d_{1,2}
$$

The solution of the cubic Equation 27 gives three real roots, then there are three eigenvalues $\lambda_{1}, \lambda_{2}, \lambda_{3}$ of the matrix $\mathrm{A}$, which are the squares of circular frequencies of bending vibrations $\omega_{m n}^{2}$ the considered a straight section of the pipeline from the waveforms at $\mathrm{m}=1,2,3$.

Equation 24 is obtained for the section of the pipeline from the hinged end section.Frequency analysis of the obtained equations for various $m$ and $n$ are the wave numbers, it will be held in later works.

\section{Conclusion}

Based on the geometrically nonlinear variant of semi-momental theory of cylindrical shells solved the problem of determining the frequency of free vibrations of a thin-walled semiunderground pipeline of large diameter, taking into account attached ground mass. The solution take into account the impact of the internal operating pressure, the geometric characteristics of the parameter of the longitudinal compressive force, and the influence of inhomogeneous elastic resistance on the ground on cross-section of the pipeline.

The results obtained in the solution can be used at the design stage of thin-walled semiunderground main gas pipelines of large diameter, and in technical expertise.

The term takes into account the impact of attached mass of ground is taken into account in the denominator of (24). Analysis of solutions shows that, depending on the attached ground mass coefficient values capable of reducing the frequency of free vibrations of semi-underground pipeline by $30-50 \%$.

The corresponding results in this article were obtained during the execution of the state task of the Ministry of Education and Science of Russia.

\section{References}

1. V. G. Sokolov, Prom. i grazhdansk.Stroit., 8 (2010)

2. A. A. Efimov, V. G. Sokolov, Izv. vuzov, Neft i gaz.,4(2010)

3. V.G. Sokolov, I.O. Razov, Vest.grazhd.Inzh.1, 36 (2013)

4. I.O. Razov, Vest. grazhd. Inzh.4, 39 (2013)

5. I.O.Razov, V.G.Sokolov, Y.V.Ogorodnova, MATEC, 73 (2016)

6. G.V. Denisov, Nauch. vest. Voronezhsk.GASU.1, 41 (2016)

7. V.V. Lalin, G.V. Denisov, Scientific Herald of the Voronezh SUACE 3, 23 (2014)

8. V.Lalin, V.Rybakov, A.Sergey, App. Mech. and Mat., 578 (2014)

9. S.V Lilkova-Markova,D.S. Lolov, Sadhana29, 3. (2004)

10. T.V. Maltseva, S.M. Dorofeev, T.V. Saltanova, Izvest.VUZ. Neft i gaz,1(2012)

11. A.V. Yavarov, G.S. Kolosova, V.V. Kuroedov,SUZiS1, 6 (2013)

12. A.V. Yavarov, Elektr nauchn zhurnal Neftegazovoe delo, 3 (2012)

13. P. Malekzadeh, M. Faridb, P. Zahedinejad, G. Karami,Journ. of Sound and Vibr. 313, 3-5 (2008)

14. B. Sobhani Aragha, A. Zeighami, M. Rafie, M.H. Ya, M. Abdel, App. Math. Modelling, 37(2013)

15. V.G. Sokolov, I.O. Razov,Vest. grazhd. Inzh. 6, 59 (2016) 
16. Haryadi Gunawan, Takashi Mikami, Shunji Kanie, Motohiro Sato,Comp. \& Struct.83, 21-22 (2005)

17. F.M. Mustafin, Neftegazovoe delo, 1 (2003)

18. E.L.Aksel'rad, V.P.Il'in, Raschet truboprovodov (Mashinostroenie, Moscow,1992)

19. X.M. Mushtari, K.Z. Galimov,Nelinejnaja teorija uprugih obolochek (Tatknigoizdat, Kazan, 1957)

20. Russian Standard SNiP 2.05.06-2010

21. V. A. Postnov, V. S. Kalinin, Vibratsiya korablya (Sudostroenie, Rostovets,1983) 